\title{
Hepatocellular Carcinoma in Asia: A Challenging Situation
}

\author{
Wasim Jafri ${ }^{1}$, Muhammad Kamran ${ }^{2}$
}

\begin{abstract}
Amongst the primary tumors of the liver, hepatocellular carcinoma (HCC) is the most common. It is also one of the most prevalent types of cancers in Asia. Mostly, HCC occurs on a background of chronic liver disease and liver cirrhosis; however, de novo HCCs can also arise in apparently normal looking livers on imaging. There are multiple risk factors for HCC, including hepatitis B and C infections, diabetes mellitus, alcohol, and nonalcoholic steatohepatitis. Other common risk factors which are known to be involved in the pathogenesis of HCC are obesity, food contaminated with aflatoxin and hemochromatosis. Many of these factors are commonly found in this part of the world, hence the high burden of disease. Besides these, smoking and familial predisposition to HCC also seem to have an important role to play in its development. Majority of HCC are missed at an early stage despite the emphasis on adequate screening and surveillance strategies. Therefore, most of the time these tumors are diagnosed at a fairly advanced stage, when palliative treatment is the only therapeutic option left. Hence, prevention of $\mathrm{HCC}$ by controlling and minimizing the possible risk factors is the need of the hour.

Keywords: Aflatoxin, Hepatitis B virus, Hepatitis C virus, Hepatocellular carcinoma, Nonalcoholic steatohepatitis.

Euroasian Journal of Hepato-Gastroenterology (2019): 10.5005/jp-journals-10018-1292
\end{abstract}

\section{INTRODUCTION}

$\mathrm{P}_{\mathrm{c}}^{\mathrm{r}}$ imary liver neoplasms include HCC, intrahepatic cholangiocarcinoma, and angiosarcoma (arising from the intrahepatic blood vessels). ${ }^{1}$ The most frequent histological type amongst these is HCC. ${ }^{1}$ Additionally, in the Asia-Pacific region, it is considered to be the third most common cause of death related to cancer. ${ }^{2}$ Worldwide, $\mathrm{HCC}$ is the sixth most common cancer, being the fifth amongst men and the seventh in women. ${ }^{3}$ The rates of incidence and mortality are comparable in HCC since most of these tumors have already progressed when diagnosed. ${ }^{4}$ A huge number of HCC cases are seen in Asian and African countries, as these regions also happen to be more prevalent in chronic infections like those with hepatitis B virus (HBV) and chronic hepatitis C virus (HCV). ${ }^{2}$ That is why the World Health Organization (WHO) has categorized chronic HBV infection and chronic HCV infection as group 1 carcinogens along with aflatoxin, a toxin which is released by Aspergillus species. ${ }^{5,6}$ This fungus grows in soil, decaying vegetation and improperly stored grains particularly in some regions of Asia where warm and humid environment prevails. ${ }^{7}$ Therefore, a high prevalence of HCC in Asia is a not so unexpected fact. Furthermore, nearly half of the cases diagnosed worldwide are reported from China only. ${ }^{8}$ In this article, we will provide an overview of the risk factors linked to HCC in Asia and the measures, in practical terms, be taken to control the disease burden of HCC in this part of the world.

\section{Risk Factors Associated with HCC in Asia}

$\mathrm{HCC}$ is an intricate disease consisting of multiple causative aspects and many risk factors. ${ }^{9,10}$ The most well-known among these are chronic infection with hepatitis B or C virus, alcohol, diabetes mellitus, and nonalcoholic steatohepatitis. ${ }^{11,12}$ Chronic viral hepatitis is a causative factor for HCC, particularly Asian endemic regions. ${ }^{13,14}$ Other risk factors for the development of HCC include intake of food-soiled with aflatoxin (as discussed above), obesity and hereditary conditions like hemochromatosis. Besides these, various metabolic disorders which include a-1 antitrypsin deficiency, while conditions like tyrosinemia and several porphyrias have also been shown to lead to HCC. ${ }^{9,11,15}$ However, these are rare disorders and therefore contribute little risk at the population level as far as HCC is concerned.
${ }^{1}$ Department of Medicine, Aga Khan University, Karachi, Pakistan

${ }^{2}$ Department of Medicine, Baqai Medical University, Karachi, Pakistan

Corresponding Author: Wasim Jafri, Department of Medicine, Aga

Khan University, Karachi, Pakistan, e-mail: wasim.jafri@aku.ed

How to cite this article: Jafri W, Kamran M. Hepatocellular Carcinoma in Asia: A Challenging Situation. Euroasian J Hepatogastroenterol 2019; 9(1):27-33.

Source of support: Nil

Conflict of interest: None

\section{Hepatitis B virus (HBV) infection and HCC}

As per the WHO fact sheet of July 2018, approximately 257 million people are infected with chronic HBV across the globe. Of these, most belong to HCC high-risk areas of Asia-Pacific and sub-Saharan Africa. ${ }^{16}$ At present, nearly three-fourths of the entire world's population of $\mathrm{CHB}$ carriers live in Asia. ${ }^{17}$ Worldwide, $50-80 \%$ of cases of HCC are thought to occur secondary to chronic HBV infection. ${ }^{18}$ Additionally, in HBV endemic or hyperendemic areas, $75-90 \%$ of cases of HCC are directly caused by chronic HBV. ${ }^{18}$

It is a well-known fact that chronic HBV infection promotes carcinogenesis in the liver as a result of damage to liver parenchyma due to inflammation. ${ }^{18}$ The presence of hepatitis B surface antigen $(\mathrm{HBsAg})$ alone without underlying cirrhosis increases the relative risk of developing HCC up to 10-30 times. ${ }^{19,20}$ Even in subjects who were seronegative for HBsAg, HBV DNA sequences have been identified in $10-20 \%$ of HCC tumors. ${ }^{21}$ The reason for this phenomenon is most likely the fact that HBV DNA gets assimilated into the genome of hepatocytes in most HCC cases who are current HBV carriers or have been so at some point in time of their lives. ${ }^{18}$

The possibility of HCC among chronic carriers of HBV also depends upon other factors. One of them is serum HBV DNA levels (viral load). One population-based study revealed that high-level viremia $(>2,000 \mathrm{IU} / \mathrm{mL}$ ) poses a greater risk of developing HCC than in than those with lower levels, irrespective of hepatitis Be antigen (HBeAg) positivity, serum alanine aminotransferase (ALT) levels, and even cirrhosis of the liver. ${ }^{22}$ Another factor with regards to the association of HBV and HCC is the genotype of the patient. Many population-based studies have shown genotype $C$ to be associated

(c) The Author(s). 2019Open Access This article is distributed under the terms of the Creative Commons Attribution 4.0 International License (https://creativecommons. org/licenses/by-nc/4.0/), which permits unrestricted use, distribution, and non-commercial reproduction in any medium, provided you give appropriate credit to the original author(s) and the source, provide a link to the Creative Commons license, and indicate if changes were made. The Creative Commons Public Domain Dedication waiver (http://creativecommons.org/publicdomain/zero/1.0/) applies to the data made available in this article, unless otherwise stated. 
with a higher risk of HCC, out of the eight main HBV genotypes $(\mathrm{A}-\mathrm{H}) .{ }^{23}$ Furthermore, data from recent studies also show that high serum HBsAg levels are also associated with an increased risk of HCC. This is especially common for those patients who have intermediate levels of HBV DNA in their blood. ${ }^{24}$

\section{Hepatitis C Virus Infection and HCC}

First discovered in 1989, hepatitis C virus (HCV) is a major cause of chronic liver disease, with over 170 million people affected across the globe. ${ }^{25}$ Although the prevalence may be decreasing in the developing countries due to increased awareness and proper hygienic conditions, it remains highly prevalent $(>3.5 \%$ estimated) in Central and East Asia, North Africa and the Middle East. ${ }^{26}$ Furthermore, due to the lack of availability of high-quality epidemiological data from the developing world, it is difficult to forecast the disease burden related to HCV infection. One of the most significant risk factors for the development of HCC in this part of the world is chronic infection with chronic HCV. Being an RNA virus, the life cycle of HCV is cytoplasmic, in contrast, hepatitis $B$ virus, where direct integration into the genetic material of the host cell takes place. ${ }^{27} \mathrm{HCV}$-associated hepatocarcinogenesis is multifactorial. There can be direct viral oncogenic effects or indirect mutagenic insults to the hepatocyte genome due to inflammation and resultant fibrosis. ${ }^{27} \mathrm{HCV}$ infection alone can predispose an individual to an increased risk of HCC development. ${ }^{28}$ As liver fibrosis advances, the risk of HCC gradually escalates. The incidence of $\mathrm{HCC}$ is approximately $1-7 \%$ per year in patients with established fibrosis. That said, HCC can, albeit rarely, develop in less fibrotic livers. ${ }^{29}$

The world is hopeful that with the discovery of highly effective direct-acting antivirals (DAAs) for HCV, there would be a decrease in HCV-related HCC. ${ }^{30}$ However, the threat is not reduced even after $\mathrm{HCV}$ eradication, and this is especially true for those patients who already have developed advanced liver fibrosis. ${ }^{31}$

$\mathrm{HCV}$, being an RNA virus (as stated above), divides in the cytoplasm of the cell so does not integrate into the genome of the host. However, this virus controls some molecular pathways that regulate the cell cycle. These include pathways that govern proliferation $^{32}$ and tumor suppressor gene p53 disruption. ${ }^{33}$ In addition, it also participates in triggering the expression of several members of the PKC superfamily, resulting in the activation of STAT3 by enhancing its phosphorylation, and hence cell transformation and apoptosis is deregulated. ${ }^{34}$

Pakistan has one of the highest prevalence of HCV among different countries in the Asia Pacific region (approximately 6\%).35 It has been estimated that around $54 \%$ of HCC in Pakistan is attributable to chronic HCV infection. ${ }^{36}$ The dominant genotype in Pakistan is genotype $3 .^{37}$

Risk of development of HCC in patients with HCV-related liver cirrhosis has been estimated by using several invasive and noninvasive models based on clinical variables. Having said that, none of these could make their place in routine clinical practice due to their limited scope of performance. ${ }^{38}$

\section{Impact of Diabetes Mellitus, Nonalcoholic Fatty Liver Disease and Obesity on HCC}

DM is an intricate metabolic state which is defined by the level of hyperglycemia resulting from defective insulin secretion, insulin action or both, ultimately leading to vascular complications. ${ }^{39}$ Although the prevalence of diabetes is increasing across the globe, it is more so in Asia. It has been estimated that 366 million individuals worldwide have this deadly illness, with $36 \%$ of those affected live in the Western Pacific region, a sizable population being in East Asia. ${ }^{40} \mathrm{DM}$ alone has been linked to approximately two to four times increased risk of HCC in some western studies. ${ }^{41}$ Similar results have been found in Asians. ${ }^{42}$

DM and liver disease are related in many ways. DM contributes toward more advanced liver fibrosis along with HCC and is associated with poor overall outcomes. ${ }^{43} \mathrm{HCC}$ has known to recur in patients with DM even after curative therapy, irrespective of the underlying etiology of the liver disease. ${ }^{43}$ In a vast majority of patients, cirrhosis itself is associated with impaired glucose tolerance (IGT). ${ }^{44}$ Twenty percent of cirrhotics have frank DM, while patients with cirrhosis of the liver suffer from insulin resistance even without DM. ${ }^{45}$

Overall, type $2 \mathrm{DM}$ is more often associated with chronic HCV infection than the general population. ${ }^{46}$ A study from Pakistan demonstrated insulin resistance in $51 \%$ of such patients. ${ }^{47}$ Another study from this part of the world showed that HCV genotype 3 infection, as well as higher body weight and age of an individual, are independent risk factors of type $2 \mathrm{DM}$ in HCV positive patients, along with a statistically important association of cirrhosis seen with type $2 \mathrm{DM}^{48}$ Kuske et al. also stressed the role of diabetes mellitus as a risk factor for HCC in patients with $\mathrm{HCV} .{ }^{49}$ Fujino et al. in a prospective study found that after adjustments for confounding factors like cigarette smoking, alcohol consumption, and other comorbid illnesses, there was an almost 2-fold relative risk (RR) of DM for primary liver cancers. ${ }^{50}$ Also, as high as $50 \%$ of patients with HCC not related to chronic HBV or HCV have DM in Pakistan. ${ }^{51}$ Hence, it would not be wrong to say that DM truly increases the risk of HCC in individuals without other contributing factors.

Nonalcoholic fatty liver disease (NAFLD) is commonly found in patients with DM. ${ }^{52}$ NAFLD is defined as the presence of fat in the liver after other contributory factors for deposition of fat have been excluded, e.g., certain drugs or significant alcohol consumption. The clinical spectrum of this disease ranges from relatively innocuous conditions like simple steatosis (nonalcoholic fatty liver; NAFL) to more sinister pathologies such as steatosis with necroinflammatory changes (nonalcoholic steatohepatitis; NASH), advanced fibrosis, cirrhosis and eventually HCC. ${ }^{52}$

NAFLD is becoming a growing problem in Asia. ${ }^{53}$ This is predominantly due to changing the socioeconomic landscape in this part of the world seen with rapid modernization and increasing wealth, leading to a more inactive lifestyle and dietary adaptations predisposing to obesity. Therefore, it can very well be stated that metabolic syndrome's hepatic manifestation is NAFLD. Also, NAFLD poses tremendous fiscal effects as far as the healthcare costs and resource consumption are concerned, as this epidemic is fast becoming the leading cause of liver cirrhosis worldwide. Chronic liver disease related to NASH may soon become the foremost cause of liver transplantation. ${ }^{54} \mathrm{~A} 5$-year case series from India elucidated that NASH-related cirrhosis was identified in 47 cases out of the 372 patients who underwent liver transplantation of which $17 \%$ had NASH-related HCC. ${ }^{55}$ Another Malaysian cross-sectional study showed similar results, where $16.4 \%$ of the 348 HCC cases were thought to arise from an unknown cause, which were presumed by the investigators to be related to NAFLD since the majority of the subjects had long-standing DM. ${ }^{56}$

There is evolving evidence to suggest that NASH-related HCC can also have de novo development in a noncirrhotic liver, which is of growing concern. Yasui et al. studied 87 patients with NASH who went on to develop HCC, of which almost half did not have biopsy proven liver cirrhosis. ${ }^{57}$ Therefore, a lot is yet to be discovered about this growing epidemic of NAFLD/ NASH and its relationship with HCC. 
Obesity is a condition in which there is a surplus of fat in the body. WHO recommends body mass index (BMI, weight in kilograms divided by height in meters squared) to classify body weight. ${ }^{58}$ The current cutoff points recommended by the WHO for being overweight and for being obese is $\geq 25 \mathrm{~kg} / \mathrm{m}^{2}$ and $\geq 30 \mathrm{~kg} / \mathrm{m}^{2}$, respectively. ${ }^{58}$ However, there has been a constant deliberation about using these proposed BMI cutoff points in Asians. The reasons for this are mainly two. Firstly, the prevalence of type 2 DM and cardiovascular risk factors in these populations is high despite having a BMI lower than $25 \mathrm{~kg} / \mathrm{m}^{2}$, and secondly, there is a sizable discrepancy between BMI, distribution of body fat and its percentage in different Asian population groups. ${ }^{58}$ Therefore, BMI cutoffs recommended by the International Association for the Study of Obesity (IASO), the International Obesity Task Force (IOTF) and the WHO for Asian adults is $23.0-24.9 \mathrm{~kg} / \mathrm{m}^{2}$ for being overweight and $\geq 25.0 \mathrm{~kg} / \mathrm{m}^{2}$ for obesity. ${ }^{59}$ Obesity and DM, along with its hepatic element of NASH, has been closely linked to metabolic syndrome with additional risks of cardiovascular disorders and stroke.

Obesity, in general, is associated with an increased relative risk (RR) of cancer-related death. Breast, colorectal, endometrial, renal cell carcinoma and esophageal adenocarcinoma have been linked to obesity. ${ }^{60}$ Obesity is also an independent predictor for HCC. This has been elucidated in both general populations as well as cirrhotic patients group by several epidemiological studies. ${ }^{61}$ In a study done on Japanese patients with decompensated cirrhosis, it was noted that higher the BMI, greater the HCC risk. ${ }^{62}$ Of particular interest are some studies which have demonstrated a combined influence of obesity and DM on HCC in the absence of chronic HBV and HCV infection. ${ }^{63}$ This effect gets even sturdier in patients who already have either viral hepatitis B or C. ${ }^{64}$ Obesity, like DM, has the potential to cause inflammation of hepatocytes, which leads to series of events such as oxidative stress and lipid peroxidation of the intracellular membranes, as a result of which there are cellular injury and necrosis, and finally $\mathrm{HCC}^{64}$

\section{Role of Aflatoxin as a Cause of HCC}

As discussed in the introductory section of this review, Aflatoxin is a metabolite of fungi of the Aspergillus species (A. flavus and $A$. parasiticus). ${ }^{7}$ It grows freely on foods such as corn, peanuts, etc. which are stored under environment conducive for its growth, for e.g., warm, moist conditions. ${ }^{7}$ This is a common site of storing many agricultural commodities in many marketplaces in the third world countries like Pakistan, India, and Bangladesh, especially in the months following the monsoon. ${ }^{7}$ There have been various studies from low-income countries which have shown their population to be chronically exposed to heavy amounts of aflatoxin in their environment. ${ }^{65}$ Of particular interest is the region of sub-Saharan Africa and southern China, where levels are the highest, especially among males. Aflatoxins are potent carcinogens which have been implicated in the development of HCC. Out of the four main aflatoxin types B1, B2, G1 and G2, the most carcinogenic potential has been found to be that of aflatoxin B1 (AFB1). ${ }^{66}$ This effect seems to be a cumulative process, as AFB1 deposits have been found in biopsy specimens of liver tissue with HCC, even when the levels of this toxin were not detectable in the serum. ${ }^{67}$ It is, therefore, thought that aflatoxin may have a contributory role to play in up to one-third of all global HCC cases. ${ }^{68}$

Although aflatoxin alone is a carcinogenic substance, the risk of $\mathrm{HCC}$ markedly escalates when concomitant chronic HCV infection is present. ${ }^{69}$ Similarly, it also appears to have a synergistic effect on HBV-induced liver cancer. ${ }^{70}$

\section{Smoking and HCC}

Across the globe, cigarette smoking is the top-most cause of preventable disease and death. Heart disease, stroke, and lung cancer are more likely to develop in this population as opposed to non-smokers. Development of many of the cancers, including HCC, is attributable to smoking. ${ }^{71}$ Chemicals in the tobacco smoke get metabolized in the liver, with some of them becoming activated as carcinogens. This effect was shown in a large retrospective study from China and was seen in both males as well as females. ${ }^{72}$ Having said that, there have been varying findings in studies among similar populations, and the true relationship between tobacco and HCC is hard to outline especially in the presence of other confounding factors like alcohol. However, more than a decade ago, it was established by the International Agency for Research on Cancer (IARC) that there was satisfactory evidence to support the fact that tobacco smoking is an independent risk factor for liver $\mathrm{HCC}^{73}$

\section{Alcohol and HCC}

According to $\mathrm{WHO}$, approximately $80 \%$ of deaths from liver disease are secondary to alcohol use. ${ }^{74}$ The International Agency for Research on Cancer (IARC), which is a part of WHO, has lately classified ethanol, acetaldehyde, and alcohol as carcinogens. ${ }^{75}$ Alcohol consumption increases the risk of various types of cancer like that of the oral cavity, head and neck, esophagus, colon and, understandably, liver. ${ }^{76}$ Alcohol, like NAFLD, can cause a wide range of hepatic injury, ranging from mild biochemical damage to alcoholic hepatitis, fatty liver, and eventually cirrhosis. The development of cirrhosis can result in HCC, which is the indirect effect of alcohol. However, alcohol can also behave as direct carcinogen due to its genotoxic effect. ${ }^{77}$ The occurrence of HCC correlates directly with the amount of alcohol consumed and is more in subjects with concomitant DM or chronic viral hepatitis $\mathrm{B}$ or $\mathrm{C}^{66}$

Association of alcohol and HCC has been well studied in Asia, with studies mainly including large cohorts. According to the Korean Cancer Prevention Study, there is a RR for HCC of 1.5 (95\% confidence interval, $\mathrm{Cl}, 1.2-2.0)$ in alcohol users as compared to non-drinkers. ${ }^{78}$ In Mongolia, for instance, one of the factors contributing to a very high risk of $\mathrm{HCC}$ is the high alcohol consumption among Mongolian men. ${ }^{79}$ Therefore, it is a matter of great concern that increasing alcohol consumption among Asian population is contributing enormously to the burden of HCC in this part of the world.

\section{Genetics and HCC: The Asian Perspective}

Besides a number of environmental risk factors discussed above, there have been reports of HCC having familial aggregation. This clustering can be explained partly due to familial transmission of $\mathrm{HBV}$ and HCV along with potentially harmful lifestyle behaviors, such as tobacco smoking and substantial alcohol consumption. This phenomenon has been frequently witnessed in eastern Asian countries highly prevalent in chronic HBV infection such as China. ${ }^{80}$ Moreover, genotype D is the predominant HBV genotype in South Asia especially India and Pakistan, and it also happens to be the most common genotype in $\mathrm{HBV}$-associated $\mathrm{HCC}$ in various series. ${ }^{81}$ However, there have been cases where there was a familial risk of HCC even among individuals who did not have evidence of chronic viral hepatitis B or C, and this has been concluded mainly from studies in the western world. ${ }^{82}$ Asians have a strong genomic predisposition for type $2 \mathrm{DM}$, which, in turn, is a risk factor for the development of HCC (as discussed above). Familial clustering of HCC was also shown in a recent case report from India where the diagnosis of HCC was confirmed by fine needle aspiration cytology (FNAC) on noncirrhotic livers without underlying chronic hepatitis $B$ or $C{ }^{83}$ 
Additionally, there are some inherited metabolic disorders which can lead to HCC. Hereditary hemochromatosis is an autosomal recessive disorder of pathological iron overload. This results in disproportionate deposition of iron in hepatic cells and those of the various body organs, namely heart, pancreatic tissue, joints, and pituitary gland, eventually leading to cirrhosis and HCC. Recent studies have suggested that the increased overall risk of HCC is around 20 -fold, with a 10 -year risk of $6 \%$ in men and $1.5 \%$ in women. ${ }^{84}$

\section{HCC prevention in Asia: What can be the Potentially Protective Targets?}

Presently, there are many treatments available for HCC. These range from curative therapies including liver resection, transplantation, percutaneous ethanol injection (PEI) and radiofrequency ablation (RFA), to palliative approaches comprising transarterial chemoembolization (TACE) and targeted oral tyrosine kinase inhibitors (e.g., sorafenib, regorafenib, and lenvatinib). ${ }^{85}$ Fairly recently, microwave ablation and stereotactic body radiation therapy are also gaining popularity, either used alone or in combination with other already established treatment modalities. ${ }^{86,87}$ However, currently available therapies are inefficient most of the time, primarily due to late tumor diagnosis. Hence, by the time the tumor is diagnosed, many patients are already beyond the Milan criteria, and increased rates of recurrence have been reported postsurgical resection. For this reason, prevention of HCC by controlling the possible risk factors is imperative.

As a general rule, prevention of HCC can be broadly classified into two types: (1) primary preventive measures that are geared towards halting HCC development in patients who have chronic liver disease secondary to any cause, and (2) secondary preventive strategies designed to prevent disease recurrence, as well as inhibition of new HCC lesions after successful HCC treatment, be it surgical or non-surgical. ${ }^{88}$ Importance of utilizing imaging and tumor markers for vigorous HCC screening and surveillance among subjects with underlying liver cirrhosis cannot be undermined. In this section, we will discuss how the target of primary prevention in HCC can be accomplished by taking various measures at the population level, and what has been done so far in this regard.

\section{Preventing HCC Related to Chronic HBV}

A major milestone in the prevention of chronic $\mathrm{HBV}$ and subsequently HBV-related HCC is the universal vaccination of infants at a large population level. This step has shown to be very impactful in halting vertical transmission (from infected mother to neonate). ${ }^{89}$ The first vaccine for HBV was developed from human serum, and it was licensed in the United States Food and Drug Administration (FDA) in $1981 .^{90}$ Later, in 1984, the recombinant yeast vaccine was introduced, which is given in three doses as intramuscular injections, and proves to have an approximately $95 \%$ efficacy. ${ }^{91}$ Many countries across the globe have since adopted the universal HBV vaccination model as their national program. This, by markedly reducing the proportion of new $\mathrm{HBV}$ infections (including adult infections), has led to a significant drop in the risk of HBVassociated HCCs. ${ }^{92}$ This phenomenon was very well depicted by a Taiwanese study, which showed a decline in the incidence of HCC among children 6-19 years of age after the nation implemented countrywide HBV vaccination in $1984 .{ }^{93}$ However, we need more time to make more definitive conclusions, as this vaccination program was familiarized to the world between 1982 and 1990, while most cases of HCC arise after 40 years of age.
There is enough evidence to support the fact that effective treatment of chronic hepatitis B with either injectable pegylated interferon-alpha (PEG-IFN-a) or oral nucleoside and nucleotide analogs (NAs) can reduce the risk of HCC in patients with early or even advanced liver fibrosis. In a study conducted in China, it was revealed that the group of chronic HBV patients receiving lamivudine had a substantively reduced incidence of HCC as opposed to those getting placebo treatment (7.4\% vs. $3.9 \%){ }^{94}$ However, cirrhotic patients on interferon therapy are at considerable risk of decompensation in terms of liver disease; therefore cautious assessment is needed before commencing injectable therapy in such individuals.

\section{HCC Prevention in Cases with Chronic HCV}

Primary prophylaxis in the form of the vaccine is not available against hepatitis $C$ virus (as opposed to hepatitis $B$ against which we have a highly efficacious vaccine). Until a few years ago, the standard management for treating chronic hepatitis $C$ was PEG-IFN- $a$ and ribavirin. However, only 50 to $60 \%$ of patients could achieve sustained virological response (SVR), that is, undetectable HCV RNA 6 months after completion of antiviral therapy. ${ }^{95}$ Furthermore, the fundamental hepatitis $C$ antiviral long-term treatment against cirrhosis (HALT-C) trial also elucidated that there remained a risk, albeit small, of developing HCC even after achieving SVR (adjusted cumulative incidence of HCC being $1.1 \%$ among those who achieved SVR, while $5.5 \%$ and $8.8 \%$ for those who either had breakthrough or relapse after initial response, and non-responders, respectively). ${ }^{96}$ Although the anti-tumor effects of interferon are well known, recent data advocates that clearance of viremia is the most important factor responsible for this risk reduction.

There was a paradigm shift in the management of chronic HCV infection with the introduction of DAAs in 2013. The SVR rates with some of these new drugs have substantially improved to more than $95 \%$, thus improving overall liver function, as well as rescuing patients from needing liver transplantation. ${ }^{97}$ Hence, it would probably not be incorrect to state that the elimination of chronic HCV with the help of DAAs would be the most effective way of preventing HCV-associated HCC. In 2016, it was somewhat disconcerting to witness some literature reporting surprisingly higher rates of de novo primary liver cancer in patients who had already achieved an SVR with DAA therapy, as well as HCC recurrence after curative treatment for early HCC. ${ }^{98,99}$ However, these studies were mostly retrospective or observational (with no control groups) and lesser sample size.

On the other hand, very large cohort studies published subsequently did not find a considerable difference in the development of HCC after antiviral treatment with DAAs. These studies included patients with both early and advanced liver fibrosis. ${ }^{100,101}$ Therefore, it can be concluded that even after the achievement of SVR, the risk of de novo HCC development cannot be entirely eliminated, although it can very well be reduced to a greater extent.

The use of DAAs for the management of HCV is relatively recent. We will be needing more evidence to show that effective treatment of HCV by DAAs will eventually result in decreasing the risk of developing $\mathrm{HCV}$ related $\mathrm{HCC}$. These medications are currently very expensive, however, some of these DAAs are available at a much cheaper price in few Asian countries (including Pakistan) as compared to the West. Nevertheless, their price will further come down as more pangenotypic drugs are been introduced in the 
market. We, therefore, hope that with the general accessibility of these drugs, the overall incidence of HCV-related HCC should decline considerably.

\section{Role of Lifestyle Modifications, Environment, and HCC Prevention}

As an emerging concept, diet and lifestyle play an important part in HCC development, even in Asian countries. Although some specific nutrients are thought to intensify HCC risk, diets rich in energy further escalate the problem as a whole. Studies have shown a positive relationship between increase dietary glycemic burden and $\mathrm{HCC}$ in patients with chronic HBV or HCV infection. ${ }^{102}$ The mainstay of NASH treatment also include diet and lifestyle changes, which, as discussed above, is pre-carcinogenic. Recent evidence suggests that adhering to a healthy diet delays HCC development to some extent in high-risk populations. ${ }^{103}$ This includes eating more fruits, vegetables, and fiber, as well as lowering the intake of dietary sugar and saturated fat.

Evidence has also shown that consistent exercise helps overcome the detrimental effects-linked to increased energy-rich diet consumption, which includes obesity and insulin resistance. Furthermore, performing at least 15 minutes of daily exercise diminish all-cause, and particularly, cancer-related mortality. ${ }^{104}$ This particular aspect of physical activity has inspired increasing attention in this area of research. Population-based studies have shown that physical activity decreases the risk of certain cancers especially esophageal, colon, lung, breast, etc. In a recent Taiwanese study, it was witnessed that the decline in risk of HCC correlated with the degree of physical activity. ${ }^{105}$ Even without weight loss, regular physical activity has been shown to reduce liver steatosis and as a result, improve insulin sensitivity.

Of late, there has been growing interest in the protective effect of coffee against HCC, especially in this part of the world. A large meta-analysis consisting of 16 studies suggested that intake of coffee is associated with decreased risk of HCC. ${ }^{106}$ The effect of coffee seems to be related to the amount consumed, irrespective of the underlying cause of liver cirrhosis. Additionally, coffee intake might indirectly modify the risk of HCC by reducing the risk of advanced fibrosis and cirrhosis. ${ }^{107}$ Besides coffee, increased fish and vitamin $\mathrm{E}$ intake have also been found to be useful in alleviating the risk of HCC. ${ }^{108}$

Keeping food products in dry storage conditions is an important step in preventing contamination from aflatoxin. Also, the use of tobacco products including cigarette smoking should be completely banned due to their strong association with HCC in addition to other cancers.

\section{ConcLusion}

HCC is a problem of great magnitude across the world, and more so in Asia due to the many risk factors which are relatively unique to this part of the globe. The overall prognosis of HCC is poor. It is, therefore, imperative that efficient and multidisciplinary efforts be taken at both national and regional levels to create awareness of this issue among at-risk populations as well as to ensure preventive measures against it so that the disease can be eliminated from Asia in particular and the world in general.

\section{References}

1. Stuver S, Trichopolous D. Cancer of the liver and biliary tract. In: Adami HO, Hunter D, Trichopolous D, editors. Textbook of cancer epidemiology. 2rd ed. New York: Oxford University Press; 2008 308-332.

2. Zhu XR, Seto WK, et al. Epidemiology of hepatocellular carcinoma in the Asia-pacific region. Gut and Liver 2016;10(3):332-339.

3. Ferlay J, Shin HR, et al. Estimates of worldwide burden of cancer in 2008: GLOBOCAN 2008. Int J Cancer 2010;127(2):2893-2917.

4. Yang JD, Roberts LR. Hepatocellular carcinoma: A global view. Nat Rev Gastroenterol Hepatol 2010;7(8):448-458.

5. IARC. Hepatitis viruses. IARC Monogr Eval Carcinog Risks Hum 1994;59.1-255.

6. IARC. Some traditional herbal medicines, some mycotoxins, naphthalene and styrene. IARC Monogr Eval Carcinog Risks Hum 2002;82:1-556.

7. Nisa $A$, Zahra $N$, et al. Detection of aflatoxins in rice samples. Bangladesh J Sci Ind Res 2014;49(3)189-194.

8. Lai CL, Ratziu V, et al. Viral hepatitis B. Lancet 2003;362(9401):20892094.

9. Gomaa Al, Khan SA, et al. Hepatocellular carcinoma: epidemiology, risk factors and pathogenesis. World J Gastroenterol 2008;14(27):43004308.

10. Di Bisceglie AM. Epidemiology and clinical presentation of hepatocellular carcinoma. J VascInterv Radiol 2002;13(9 Pt 2):S169S171.

11. El-Serag HB, Rudolph KL. Hepatocellular carcinoma: epidemiology and molecular carcinogenesis. Gastroenterology 2007;132(7):25572576.

12. Poon D, Anderson BO, et al. Management of hepatocellular carcinoma in Asia: consensus statement from the Asian Oncology Summit 2009. Lancet Oncol 2009;10(11):1111-1118.

13. Chisari FV, Klopchin K, et al. Molecular pathogenesis of hepatocellular carcinoma in hepatitis B virus transgenic mice. Cell 1989;59(6):1145-1156.

14. Idilman R, De Maria N, et al. Pathogenesis of hepatitis B and C-induced hepatocellular carcinoma. J Viral Hepat 1998;5(5):285-299.

15. Montalto G, Cervello M, et al. Epidemiology, risk factors, and natural history of hepatocellular carcinoma. Ann NY Acad Sci 2002;963:13-20.

16. McGlynn KA, London WT. Epidemiology and natural history of hepatocellular carcinoma. Best Pract Res Clin Gastroenterol 2005;19(1):3-23.

17. Schweitzer A, Horn J, et al. Estimations of worldwide prevalence of chronic hepatitis B virus infection: a systematic review of data published between 1965 and 2013. Lancet 2015;386(10003):1546-1555.

18. Block TM, Mehta AS, et al. Molecular viral oncology of hepatocellular carcinoma. Oncogene 2003;22(33):5093-5107.

19. Mori $M$, Hara $M$, et al. Prospective study of hepatitis $B$ and $C$ viral infections, cigarette smoking, alcohol consumption, and other factors associated with hepatocellular carcinoma risk in Japan. Am J Epidemiol 2000;151(2):131-139.

20. Yang $\mathrm{HI}$, Lu SN, et al. Hepatitis B e antigen and the risk of hepatocellular carcinoma. N Engl J Med 2002;347(3):168-174.

21. Ming L, Thorgeirsson SS, et al. Dominant role of hepatitis B virus and cofactor role of aflatoxin in hepatocarcinogenesis in Qidong, China. Hepatology 2002;36(5):1214-1220.

22. Chen CJ, Yang $\mathrm{HI}$, et al; REVEAL-HBV Study Group. Hepatitis B virus DNA levels and outcomes in chronic hepatitis B. Hepatology 2009:49(5 Suppl):S72-S84.

23. McMahon BJ. Natural history of chronic hepatitis B. Clin Liver Dis 2010;14(3):381-396.

24. Tseng TC, Liu CJ, et al. High levels of hepatitis B surface antigen increase risk of hepatocellular carcinoma in patients with low HBV load. Gastroenterology 2012;142(5):1140-1149.e3.

25. Szabo E, Lotz G, et al. Viral hepatitis: new data on hepatitis C infection. Pathol Oncol Res 2003;9(4):215-221.

26. MohdHanafiah K, Groeger J, et al. Global epidemiology of hepatitis $C$ virus infection: new estimates of age-specific antibody to $\mathrm{HCV}$ seroprevalence. Hepatology 2013;57(4):1333-1342.

27. Lin MV, King LY, et al. Hepatitis C virus-associated cancer. Annu Rev Pathol 2015;10:345-370.

28. Massarweh NN, El-Serag HB. Epidemiology of Hepatocellular Carcinoma and Intrahepatic Cholangiocarcinoma. Cancer Control 2017;24(3). 
29. Yoshida $H$, Shiratori $Y$, et al. Interferon therapy reduces the risk for hepatocellular carcinoma: national surveillance program of cirrhotic and noncirrhotic patients with chronic hepatitis C in Japan. IHIT Study Group Inhibition of Hepatocarcinogenesis by Interferon Therapy. Ann Intern Med 1999;131(3):174-181.

30. Chung RT, Baumert TF. Curing chronic hepatitis C-the arc of a medical triumph. N Engl J Med 2014;370(17):1576-1578.

31. Van der Meer AJ, et al. Association between sustained virological response and all-cause mortality among patients with chronic hepatitis C and advanced hepatic fibrosis. JAMA 2012;308(24):2584-2593.

32. Munakata T,Nakamura $M$, et al.Down-regulation of the retinoblastoma tumor suppressor by the hepatitis C virus NS5B RNA-dependent RNA polymerase. Proc Natl Acad Sci U S A 2005; 102(50):18159-18164.

33. McGivern DR, Lemon SM. Virus-specific mechanisms of carcinogenesis in hepatitis C virus associated liver cancer. Oncogene 2011;30(17):19691983.

34. Li Y, Zhang Q, et al. Hepatitis C virus activates Bcl-2 and MMP-2 expression through multiple cellular signaling pathways. J Virol 2012 Dec;86(23):12531-12543.

35. Al Kanaani Z, Mahmud S, et al. The epidemiology of hepatitis $C$ virus in Pakistan: systematic review and meta-analyses. R Soc Open Sci 2018;5(4).

36. Alavian SM, Haghbin H. Relative Importance of Hepatitis B and C Viruses in Hepatocellular Carcinoma in EMRO Countries and the Middle East: A Systematic Review. Hepat Mon 2016;16(3):e35106.

37. Khan A, Tanaka Y, et al. Epidemic spread of hepatitis C virus genotype $3 a$ and relation to high incidence of hepatocellular carcinoma in Pakistan. J Med Virol 2009;81(7):1189-1197.

38. El-Serag HB, Kanwal F, et al. A new laboratory-based algorithm to predict development of hepatocellular carcinoma in patients with hepatitis C and cirrhosis. Gastroenterology 2014;146(5):1249-1255.

39. Chawla A, Chawla R, et al. Microvasular and macrovascular complications in diabetes mellitus: Distinct or continuum? Indian J Endocrinol Metab 2016;20(4):546-551.

40. International Diabetes Federation. IDF diabetes atlas, 6 th ed [Internet]. Brussels: International Diabetes Federation; c2014. Available from: http://www.idf. org/sites/default/files/EN_6E_Atlas_Full_0.pdf.

41. Davila JA, Morgan RO, et al. Diabetes increases the risk of hepatocellular carcinoma in the United States: a population based case control study. Gut 2005;54(4):533-539.

42. Ohishi W, Fujiwara S, et al. Risk factors for hepatocellular carcinoma in a Japanese population: a nested case-control study. Cancer Epidemiol Biomarkers Prev 2008;17(4):846-854.

43. El-Serag HB. Hepatocellular carcinoma: recent trends in the United States. Gastroenterology 2004 Nov;127(5 Suppl 1):S27-S34.

44. Holstein A, Hinze $S$, et al. Clinical implication of hepatogenous diabetes in liver cirrhosis. J Gastroenterol Hepatol 2002;17(6):677-681.

45. Garcia-Compean D, Jaquez-Quintana JO, et al. Liver cirrhosis and diabetes: Risk factors, pathophysiology, clinical implications and management. World J Gastroenterol 2009;15(3):280-288.

46. Vanni $E$, Abate ML, et al. Sites and mechanisms of insulin resistance in non obese, non-diabetic patients with chronic hepatitis $C$. Hepatology 2009;50(3):697-706.

47. Kiran Z, Zuberi BF, et al. Insulin resistance in non-diabetic patients of chronic Hepatitis C. Pak J Med Sci 2013;29(1):201-204.

48. Memon MS, Arain Zl, et al. Prevalence of type 2 diabetes mellitus in hepatitis $C$ virus infected population: a Southeast Asian study. J Diabetes Res 2013;2013:539361.

49. Kuske L, Mensen A, et al; Swiss Hepatitis C Cohort Study. Characteristics of patients with chronic hepatitis $C$ who develop hepatocellular carcinoma. Swiss Med Wkly 2012;142:w13651.

50. Fujino Y, Mizoue T, et al. Prospective study of diabetes mellitus and liver cancer in Japan. Diabetes Metab Res Rev 2001;17(5):374-379.

51. Butt AS, Hamid $S$, et al. Hepatocellular carcinoma in Native South Asian Pakistani population; Trends, clinico-pathological characteristics \& differences in viral marker negative \& viral-hepatocellular carcinoma. BMC Research Notes 2013;6:137.

52. Chalasani $N$, Younossi $Z$, et al. The diagnosis and management of nonalcoholic fatty liver disease: practice Guideline by the
American Association for the Study of Liver Diseases, American College of Gastroenterology, and the American Gastroenterological Association. Hepatology 2012;55(6):2005-2023.

53. Fan JG, Kim SU, et al. New trends on obesity and NAFLD in Asia. J Hepatol 2017;67(4):862-873.

54. Mikolasevic I, Filipec-Kanizaj T, et al. Nonalcoholic fatty liver disease and liver transplantation - where do we stand? World J Gastroenterol 2018;24(14):1491-1506.

55. Jain D, Nayak NC, et al. Hepatocellular carcinoma in nonalcoholic fatty liver cirrhosis and alcoholic cirrhosis: risk factor analysis in liver transplant recipients. Eur J Gastroenterol Hepatol 2012;24(7):840-848.

56. Goh KL, Razlan H, et al. Liver cancer in Malaysia: epidemiology and clinical presentation in a multiracial Asian population. J Dig Dis 2015;16(3):152-158.

57. Yasui K, Hashimoto E, et al. Characteristics of patients with nonalcoholic steatohepatitis who develop hepatocellular carcinoma. Clin Gastroenterol Hepatol 2011;9(5):428-433.

58. WHO Expert Consultation. Appropriate body-mass index for Asian populations and its implications for policy and intervention strategies. Lancet 2004;363(9403):157-163.

59. The International Association for the Study of Obesity and the International Obesity Task Force. The Asia-Pacific perspective: redefining obesity and its treatment. Australia: IASO and IOTF, 2000.

60. Calle EE, Kaaks R. Overweight, obesity and cancer: epidemiological evidence and proposed mechanisms. Nat Rev Cancer 2004;4(8): 579-591.

61. Saunders D, Seidel D, et al. Systematic review: the association between obesity and hepatocellular carcinoma - epidemiological evidence. Aliment Pharmacol Ther 2010;31(10):1051-1063.

62. Muto $Y$, Sato $S$, et al. Overweight and obesity increase the risk for liver cancer in patients with liver cirrhosis and long-term oral supplementation with branched-chain amino acid granules inhibits liver carcinogenesis in heavier patients with liver cirrhosis. Hepatol Res 2006;35(3):204-214.

63. Polesel J, Zucchetto A, et al. The impact of obesity and diabetes mellitus on the risk of hepatocellular carcinoma. Ann Oncol 2009;20(2):353-357.

64. Chen $\mathrm{CL}$, Yang $\mathrm{HI}$, et al. Metabolic factors and risk of hepatocellular carcinoma by chronic hepatitis B/C infection: a follow-up study in Taiwan. Gastroenterology 2008;135(1):111-121.

65. Williams JH, Phillips TD, et al. Human aflatoxicosis in developing countries: a review of toxicology, exposure, potential health consequences, and interventions. Am J Clin Nutr 2004;80(5):1106-1122.

66. IARC. Chemical agents and related occupations. IARC Monogr Eval Carcinog Risks Hum 2012;100F:1-628.

67. Groopman JD, Kensler TW, et al. Protective interventions to prevent aflatoxin-induced carcinogenesis in developing countries. Annu Rev Public Health 2008;29:187-203.

68. Liu Y, Wu F. Global Burden of Aflatoxin-Induced Hepatocellular Carcinoma:ARiskAssessment. Environ Health Perspect 2010;118(6):818824.

69. Kirk GD, Lesi OA, et al. 249(ser) P53 mutation in plasma DNA, hepatitis $B$ viral infection, and risk of hepatocellular carcinoma. Oncogene 2005;24(38):5858-5867.

70. Qian GS, Ross RK, et al. A follow-up study of urinary markers of aflatoxin exposure and liver cancer risk in Shanghai, People's Republic of China. Cancer Epidemiol Biomarkers Prev 1994;3(1): 3-10.

71. Koh WP, Robien K, et al. Smoking as an independent risk factor for hepatocellular carcinoma: the Singapore Chinese Health Study. Br J Cancer 2011;105(9):1430-1435.

72. Chen ZM, Liu BQ, et al. Smoking and liver cancer in China: case-control comparison of 36,000 liver cancer deaths vs. 17,000 cirrhosis deaths. Int J Cancer 2003;107(1):106-112.

73. IARC. Tobacco smoke and involuntary smoking. IARC Monogr Eval Carcinogen risks Humans 2004;83:1-1438.

74. Sheron N, Hawkey C, et al. Projections of alcohol deaths--a wake-up call. Lancet 2011;377(9774):1297-1299.

75. Testino $G$. The burden of cancer attributable to alcohol consumption. Maedica (Buchar) 2011;6(4):313-320. 
76. Secretan B, Straif K, et al. A review of human carcinogens--Part E: tobacco, areca nut, alcohol, coal smoke, and salted fish. Lancet Oncol 2009;10(11):1033-1034.

77. Donato F, Tagger A, et al. Alcohol and hepatocellular carcinoma: the effect of lifetime intake and hepatitis virus infections in men and women. Am J Epidemiol 2002;155(4):323-331.

78. Jee $\mathrm{SH}, \mathrm{Ohrr} \mathrm{H}$, et al. Cigarette smoking, alcohol drinking, hepatitis $\mathrm{B}$, and risk for hepatocellular carcinoma in Korea. J Natl Cancer Inst 2004;96(24):1851-1856.

79. Alcorn T. Mongolia's struggle with liver cancer. Lancet 2011; 377(9772):1139-1140.

80. Cai RL, Meng W, et al. Segregation analysis of hepatocellular carcinoma in a moderately high-incidence area of East China. World J Gastroenterol 2003;9(11):2428-2432.

81. Pujol FH, Navas MC, et al. Worldwide genetic diversity of HBV genotypes and risk of hepatocellular carcinoma. Cancer Lett 2009;286(1):80-88.

82. Turati F, Edefonti V, et al. Family history of liver cancer and hepatocellular carcinoma. Hepatology 2012;55(5):1416-1425.

83. Chandra S, Kusum A, et al. Familial Hepatocellular Carcinoma- First Reported Case from India. J Clin Diagn Res 2016;10(3):ED11-12.

84. Elmberg $M$, Hultcrantz $R$, et al. Cancer risk in patients with hereditary hemochromatosis and in their first-degree relatives. Gastroenterology 2003;125(6):1733-1741.

85. Kudo M. Systemic therapy for hepatocellular carcinoma: Latest advances. Cancers (Basel) 2018;10(11).

86. Facciorusso A, Di Maso M, et al. Microwave ablation versus radiofrequency ablation for the treatment of hepatocellular carcinoma: A systematic review and meta-analysis. Int J Hyperthermia 2016;32(3):339-344.

87. Jun BG, Kim SG, et al. Combined therapy of transarterial chemoembolization and stereotactic body radiation therapy versus transarterial chemoembolization for $<5 \mathrm{~cm}$ hepatocellular carcinoma: Propensity score matching analysis. PLoS One 2018;13(10).

88. Lok AS. Prevention of hepatitis B virus-related hepatocellular carcinoma. Gastroenterology 2004;127(5 Suppl 1):S303-S309.

89. Gentile I, Borgia G.Vertical transmission of hepatitis B virus: challenges and solutions. Int J Womens Health2014;6:605-611.

90. Szmuness W, Stevens CE, et al. Hepatitis B vaccine: demonstration of efficacy in a controlled clinical trial in a high-risk population in the United States. N Engl J Med 1980;303(15):833-841.

91. Komatsu $\mathrm{H}$. Hepatitis $B$ virus: Where do we stand and what is the next step for eradication? World J Gastroenterol 2014;20(27):8998-9016.

92. Luo Z, Li L, Ruan B. Impact of the implementation of a vaccination strategy on hepatitis B virus infections in China over a 20 -year period. Int J Infect Dis 2012;16(2):e82-e88.
93. Chang MH, You SL, et al. Decreased incidence of hepatocellular carcinoma in hepatitis B vaccinees: a 20-year follow-up study. J Natl Cancer Inst 2009;101(19):1348-1355.

94. Liaw YF, Sung JJ, et al. Lamivudine for patients with chronic hepatitis B and advanced liver disease. N Engl J Med 2004;351(15): 1521-1531.

95. Rosen HR. Clinical practice. Chronic hepatitis C infection. N Engl J Med 2011;364(25):2429-2438.

96. Morgan TR, Ghany MG, et al. Outcome of sustained virological responders with histologically advanced chronic hepatitis C. Hepatology 2010 Sep;52(3):833-844.

97. Charlton M, Everson GT, et al. Ledipasvir and sofosbuvir plus ribavirin for treatment of HCV infection in patients with advanced liver disease. Gastroenterology 2015;149(3):649-659.

98. Conti F, Buonfiglioli F, et al. Early occurrenceand recurrence of hepatocellular carcinoma in HCVrelated cirrhosis treated with directacting antivirals. J Hepatol 2016;65(4):727-733.

99. Reig M, Marino Z, et al. Unexpected high rate of early tumor recurrence in patients with $\mathrm{HCV}$-related HCC undergoing interferonfree therapy. J Hepatol 2016;65(4):719-726.

100. Finkelmeier F, Dultz G, et al. Risk of de novo Hepatocellular Carcinoma after HCV Treatment with Direct-Acting Antivirals.Liver Cancer 2018;7(2):190-204.

101. Mun EJ, Green P, et al. No difference between directacting antivirals for hepatitis $C$ in hepatocellular carcinoma risk. Eur J Gastroenterol Hepatol 2019;31(1):47-52.

102. Lagiou P, Rossi M, et al. Glycemic load in relation to hepatocellular carcinoma among patients with chronic hepatitis infection. Ann Oncol 2009;20(10):1741-1745.

103. Mandair DS, Rossi RE, et al. The impact of diet and nutrition in the prevention and progression of hepatocellular carcinoma. Expert Rev Gastroenterol Hepatol 2014;8(4):369-382.

104. Wen CP, Wai JP, et al. Minimum amount of physical activity for reduced mortality and extended life expectancy: a prospective cohort study. Lancet 2011;378(9798):1244-1253.

105. Wen CP, Lin J, et al. Hepatocellularcarcinoma risk prediction model for the general population: the predictivepower of transaminases. J Natl Cancer Inst 2012;104(20):1599-1611.

106. Sang LX, Chang B, et al. Consumption of coffee associated with reduced risk of liver cancer: a meta-analysis. BMC Gastroenterol 2013;13: 34.

107. Torres DM, Harrison SA. Is it time to write a prescription for coffee? Coffee and liver disease. Gastroenterology 2013;144:670-672.

108. Miyanishi K, Hoki T, et al. Prevention of hepatocellular carcinoma: Focusing on antioxidant therapy. World J Hepatol 2015;7(3): 593-599. 